\title{
Characterisation of C-F Polymer Film Formation on the Air-Bearing Surface Etched Sidewall of Fluorine-Based Plasma Interacting with $\mathrm{AL}_{2} \mathrm{O}_{3}-\mathrm{TiC}$ Substrate
}

\author{
Alonggot Limcharoen, ${ }^{1}$ Pichet Limsuwan, ${ }^{1}$ Chupong Pakpum, ${ }^{2}$ and Krisda Siangchaew ${ }^{3}$ \\ ${ }^{1}$ Department of Physics, Faculty of Science, King Mongkut's University of Technology Thonburi, Bangkok 10140, Thailand \\ ${ }^{2}$ Division of Material Science, Faculty of Science, Maejo University, Chiang Mai 50290, Thailand \\ ${ }^{3}$ Western Digital (Thailand) Company Limited, Ayutthaya 13160, Thailand \\ Correspondence should be addressed to Alonggot Limcharoen; numaoy_paditam@hotmail.com
}

Received 25 February 2013; Accepted 28 May 2013

Academic Editor: Shafiul Chowdhury

Copyright (C) 2013 Alonggot Limcharoen et al. This is an open access article distributed under the Creative Commons Attribution License, which permits unrestricted use, distribution, and reproduction in any medium, provided the original work is properly cited.

$\mathrm{C}-\mathrm{F}$ polymer redeposition is generated on the etched sidewall of the patterned air-bearing surface (ABS). This C-F polymer is a byproduct from fluorine-based plasma using a Surface Technology Systems multiplex-pro air-bearing etch (ABE). The morphology of the re-deposition and the composite element was observed by a scanning electron microscope (SEM). The chemical bonding results were characterised via X-ray photoelectron spectroscopy, attenuated total reflected infrared spectroscopy and visible Raman spectroscopy. The purpose of this work is to demonstrate a modification of $\mathrm{AlF}_{3}$ re-deposition to $\mathrm{C}-\mathrm{F}$ polymer re-deposition, which is easily stripped out by an isopropyl alcohol-based solution. The benefit of this research is the removal of the re-deposition in the resist strip process without additional cleaning process steps.

\section{Introduction}

The processes used to fabricate a recording head, also known as a slider, are composed of lapping, machining, lithography, deposition, and etching steps.

Dry etching is a key process that enables sliders to fly over media by creating a pattern on the slider. Air-bearing surfaces (ABSs) are designed to control the variable number of the positive pressure that is generated by the force pushing the slider away from the medium and the negative pressure that is generated by the force pulling the slider towards the medium. As a consequence of balancing the push and pull forces on the slider, a constant fly height can be maintained across the different surfaces: the inner diameter zone, middle diameter zone, and outer diameter zone of the medium.

Reactive ion etching (RIE) is a very suitable technology for fabricating etched ABS patterns. RIE utilises the synergistic effect of ion bombardment combined with a chemical reaction in a plasma bulk. The most commonly used RIE method is an inductive coupled plasma (ICP) system. This system provides high-density plasma that meets the requirements for processing at a high volume and high reliability in production. There are two main components of an ICP system in the etching process: the plasma source and the substrate holder. For the plasma source, a radio frequency (RF) power supply and inductive coil for plasma generation are used. The substrate holder (also called an electrode), which is biased with RF power, accommodates a wafer and causes the positive ions to directly bombard the substrate.

Since electrons are more mobile than positive ions in the plasma, a negative DC voltage is developed on the electrode where the RF power is introduced. Since the substrate is placed on this electrode, the substrate experiences the bombardment from the accelerated ions onto the electrode. The ion bombardment causes physical damage to the substrate and facilitates desorption of the chemical reaction. The combination of chemical etching and physical etching leads to the etching rate being dramatically increased [1] 
The etching of $\mathrm{Al}_{2} \mathrm{O}_{3}$ in noncorrosive plasma is very difficult. For example, when the substrate is exposed to $\mathrm{F}$ containing plasmas, $\mathrm{Al}_{2} \mathrm{O}_{3}$ reacts with $\mathrm{F}$ to form a mixture of $\mathrm{AlF}_{3}$ and $\mathrm{Al}_{x} \mathrm{O}_{y} \mathrm{~F}_{z}[2,3]$. These reaction by-products, known as RIE redeposition, are nonvolatile and are resputtered onto the substrate surface, tenaciously adhering to the sidewall. Thus, it is not expected that reactive ion etching of $\mathrm{Al}_{2} \mathrm{O}_{3}$ would occur in F-containing plasma. On the other hand, $\mathrm{TiC}$ reacting with F-containing plasma will generate $\mathrm{TiF}_{4}$, which is volatile and can be pumped out from the process chamber with a vacuum pump.

The reaction by-products, as mentioned above, are not volatile; therefore, the remaining $\mathrm{AlF}_{3}$ and $\mathrm{Al}_{x} \mathrm{O}_{y} \mathrm{~F}_{z}$ redeposition could cause reliability, performance, and durability issues in the hard disk drive. These redeposition particles have high potential to be dislodged from the ABS during a disk operation, leading to the medium and/or read-write head getting damage, which causes data loss or, worse, the complete breakdown of the disk drive [4].

Due to the RIE redeposition problem described above, we are motivated to focus on slider cleanliness, particularly the ABS sidewall of a slider after the process is completed. To remove the redeposition, a special chemical solution must be used in the conventional cleaning process. However, this particular chemical causes corrosion in the high moment metals (such as CoFe-based alloys) that are currently used in magnetic recording heads, as necessary to meet areal density requirements.

Exploring new gas chemistry for the etching process is necessary. The chlorine that is contained in the plasma will etch $\mathrm{Al}_{2} \mathrm{O}_{3}-\mathrm{TiC}$ and will produce $\mathrm{TiCl}_{4}, \mathrm{AlCl}_{3}$, and $\mathrm{Al}_{2} \mathrm{Cl}_{3}$ as by-products; these are all volatile compounds [2] that are highly corrosive to the device [5]. Fukushima et al. [6] reported that no sidewall film was observed by using $\mathrm{Cl}_{2} / \mathrm{BCl}_{3}$ and $\mathrm{Ar} / \mathrm{Cl}_{2} / \mathrm{BCl}_{3}$ plasma. Unfortunately, chlorine is well known as a corrosive agent to a magnetic recording head, so using chlorine-based plasma is not possible in the slider fabrication process.

The fluorine to carbon $(\mathrm{F} / \mathrm{C})$ ratio model is a very interesting concept. Coburn and Winters $[7,8]$ proposed the $\mathrm{F} / \mathrm{C}$ ratio model, stating that increasing the $\mathrm{F} / \mathrm{C}$ ratio within the plasma by adding $\mathrm{O}_{2}$ enhanced the etch rate of $\mathrm{SiO}_{2}$. Conversely, the plasma was decreased by adding $\mathrm{H}_{2}$ or $\mathrm{CHF}_{3}$, so the etch rate of $\mathrm{SiO}_{2}$ could be reduced. Furthermore, when the $\mathrm{F} / \mathrm{C}$ ratio was below a critical value, the plasma might stop etching and generate polymer deposition instead.

From a literature review, it is clear that the F/C ratio concept for $\mathrm{SiO}_{2}$ etching has been used in semiconductor manufacturing. Ji et al. [9] studied the variety of gas chemistry with different $\mathrm{F} / \mathrm{C}$ ratios to etch on $\mathrm{SiO}_{2}$ dielectric materials to result in a high aspect ratio. It was found that a high aspect ratio of dielectric etching was able to be achieved using gas chemistry with a lower F/C ratio because of the fluorocarbon species that fragmented from the parent molecule. These species could be generated by adding a protective polymer to the photo-resist surface, creating a sidewall polymer film and delivering fluorine to the surface region that is being etched.

Fluorine-based plasma, such as tetrafluoromethane $\left(\mathrm{CF}_{4}\right)$ plasma, has been utilised in the industry for many years to etch $\mathrm{Al}_{2} \mathrm{O}_{3}-\mathrm{TiC}$ during slider fabrication. Regarding the $\mathrm{F} / \mathrm{C}$ ratio, $\mathrm{CF}_{4}$ has a ratio of 4 , meaning that this plasma can be used to etch the substrate. Due to the by-products of etching the $\mathrm{Al}_{2} \mathrm{O}_{3}-\mathrm{TiC}$ substrate with $\mathrm{CF}_{4}$ plasma not being volatile, there is plenty of redeposition of $\mathrm{AlF}_{3}$ and $\mathrm{Al}_{x} \mathrm{O}_{y} \mathrm{~F}_{z}$ along the sidewall.

To our knowledge, the $\mathrm{F} / \mathrm{C}$ ratio concept has not been applied to the etching of $\mathrm{Al}_{2} \mathrm{O}_{3}-\mathrm{TiC}$ substrate that is used to create the ABS pattern of a slider head. For ABS etching, $\mathrm{CF}_{4}+\mathrm{H}_{2}$ plasma has been studied in the fabrication of femtomagnetic recording heads [2], but the study did not concentrate on the RIE redeposition issue discussed in this present work.

By manipulating the $\mathrm{F} / \mathrm{C}$ ratio in plasma, it is expected that the selected recipe will provide polymer redeposition $\left(\mathrm{C}_{x} \mathrm{~F}_{y}\right.$ redeposition), and it should adhere to the etched sidewall during the etching process as well. However, the polymer redeposition will be removed easier than the current RIE redeposition $\left(\mathrm{AlF}_{3}\right.$ redeposition). Common solvents such as acetone and isopropyl alcohol (IPA) should dissolve a polymer-based redeposition easily.

The purpose of this paper is to demonstrate that $\mathrm{C}-\mathrm{F}$ polymer redeposition adheres to the AlTiC ABS etched sidewall. Characterisation of the polymer redeposition was done by X-ray photoelectron spectroscopy (XPS), Fourier transform infrared microspectroscopy (ATR FTIR), visible Raman spectroscopy, and electron spectroscopy.

\section{Experiment}

To get an etching condition that provides polymer redeposition, the experiments were conducted by using the design of experiment DOE method. The key parameters were (1) coil power $(300 \mathrm{~W}-700 \mathrm{~W}),(2)$ total gas flow rate $(8 \mathrm{sccm}-$ $20 \mathrm{sccm}$ ), and (3) gas type $\left(\mathrm{CF}_{4}\right.$ and $\left.\mathrm{C}_{4} \mathrm{~F}_{8}\right)$. These parameters were studied while keeping the others constant, which were (1) platen power at $550 \mathrm{~W},(2)$ pressure at $4 \mathrm{mTorr}$, and (3) chiller temperature at $-5^{\circ} \mathrm{C}$.

To create the pattern of the etching mask on the AlTiC substrate, the wafer was processed with a conventional photolithography process. All RIE etching conditions from the DOE table were performed. The mask residue was removed using a resist strip process (IPA-based solution). The amount of RIE redeposition that adhered to the etched sidewall was observed by a scanning electron microscope (SEM). There was no redeposition residue after the resist strip process was completed. This indicated that the parts were successfully etched with conditions that provided polymer redeposition.

Then, the patterned AlTiC substrate (which had a tapered profile with the sidewall at an angle of $65^{\circ}$ ) was prepared for the polymer redeposition. The patterned AlTiC substrate was etched without a polymer mask so that the redeposition accumulated on the taper sidewall, thus avoiding system noise during XPS, ATR FTIR, and Raman analysis.

The RIE machine (STS multiplex ICP reactive ion etching) was used as the experimental machine (Figure 1). Reactive ion etching utilises the synergistic effect of ion bombardment and chemical reactions in plasma. In a conventional reactive ion system, $\mathrm{AlTiC}$, which is used as the base 


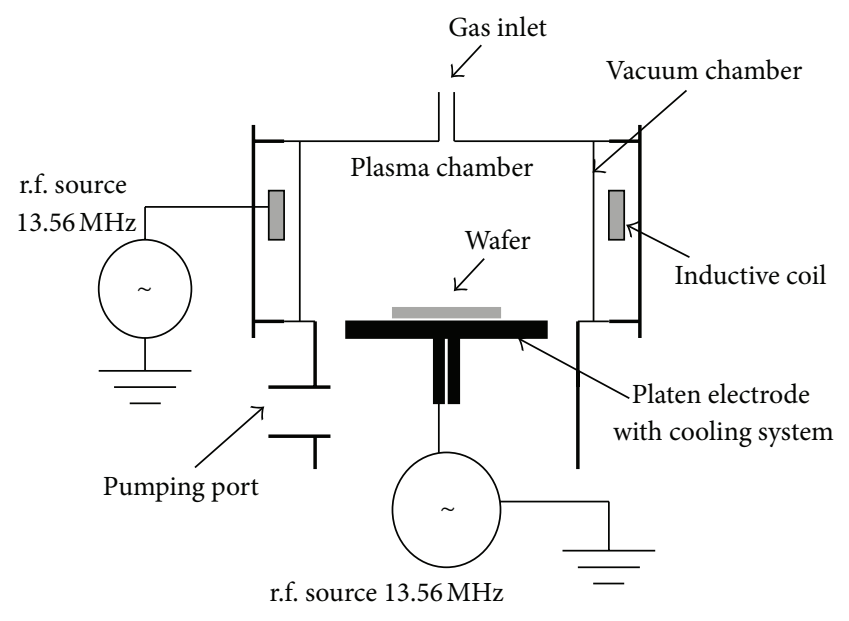

FIGURE 1: Schematic of multiplex-pro air-bearing etch ICP process chamber [10].

substrate/wafer with a patterned wet film photo resistance (with a thickness of approximately $20 \mu \mathrm{m}$ ), is placed on an electrode that is powered by a $13.56 \mathrm{MHz} \mathrm{RF}$ generator. The power is coupled into a chamber that contains a reactive gas to generate plasma. The reactive gas that was used in this experiment was octafluorocyclobutane $\left(\mathrm{C}_{4} \mathrm{~F}_{8}\right)$ with a gas flow rate of $14 \mathrm{sccm}$.

Finally, it was found that the etching conditions that provide polymer redeposition are coil power $=700 \mathrm{~W}$, platen power $=550 \mathrm{~W}, \mathrm{C}_{4} \mathrm{~F}_{8}$ gas flow rate $=14 \mathrm{sccm}$, pressure $=$ $4 \mathrm{mTorr}$, and chiller temperature $=-5^{\circ} \mathrm{C}$. The etching rate at these conditions was $57 \mathrm{~nm} / \mathrm{min}$. We prepared the polymer redeposition on a patterned $\mathrm{AlTiC}$ substrate with these etching conditions for 900 minutes to make sure that the size of the redeposition material was big enough to get a signal from the attenuated total reflected infrared spectroscopy and visible Raman spectroscopy analysis. A Philips XL 30S FEG scanning electron microscope (SEM) was used to investigate the morphology of the polymer redeposition. The composite element of the redeposition was detected by using energy dispersive X-ray spectroscopy (SEM-EDX). Raman analysis was performed under an inVia Reflex Raman microscope. This system utilises laser excitation at a wavelength of $514 \mathrm{~nm}$ and has a spectral range from $100 \mathrm{~cm}^{-1}$ to $3000 \mathrm{~cm}^{-1}$ with a resolution of $\sim 1 \mathrm{~cm}^{-1}$. For infrared analysis, the polymer redeposition spectrum was acquired by using a Nicolet 6700 ATR FTIR spectrometer within the spectral range from $650 \mathrm{~cm}^{-1}$ to $4000 \mathrm{~cm}^{-1}$ with a resolution of $4 \mathrm{~cm}^{-1}$. The chemical bonding state of the polymer redeposition was analysed by using a PHI Quantera SXM scanning X-ray microscope, with an A1K X-ray source. A beam size of $10 \mu \mathrm{m}$ with a takeoff angle of $60^{\circ}$ was used to analyse the redeposition. A curve fitting technique, which was combined with Gaussian and Lorentzian functions on a Shirley-type background, was used to assign the chemical bonding state. The percentages of the crosslink of polymer redeposition were also calculated after subtracting the signal from the AlTiC substrate.

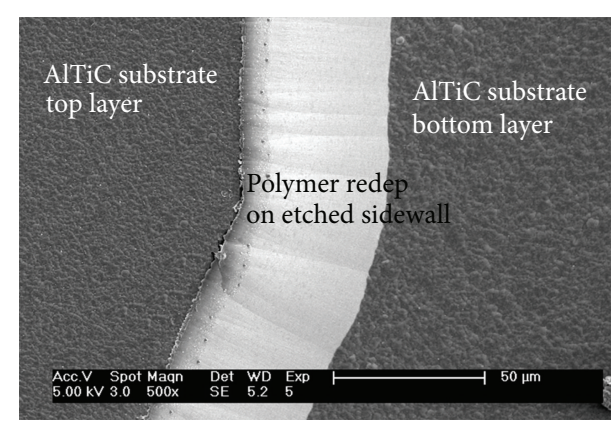

(a)

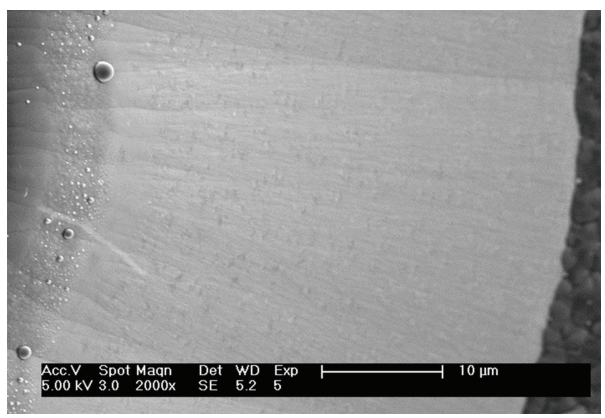

(b)

FIGURE 2: SEM image of prepared polymer redeposition. (a) Polymer redeposition is stuck along the etched sidewall. (b) Polymer redeposition surface.

\section{Results and Discussion}

3.1. Scanning Electron Microscopy (SEM). The SEM image of the prepared polymer redeposition shows that the polymer redeposition sticks along the etched sidewall, as shown in Figure 2(a). The morphology of the bulk (the redeposition material) appears ribbon-like and adheres to the sidewall, while AlTiC's surface regions of two different step heights are etched by the plasma and then present high surface roughness. The taper feature allows the redeposition material to accumulate on it. The very smooth surface of the redeposition shows a dense structure, as shown in Figure 2(b).

The SEM-EDX image in Figure 3 shows that the polymer redeposition contains carbon atoms (63.70 At\%) and fluorine atoms (34.32 At\%) as the major components.

3.2. Visible Raman Spectroscopy. Visible Raman spectroscopy is particularly sensitive to $\mathrm{sp}^{2}$ hybridised carbon configurations. Using Gaussian curves to fit the spectra, we observed two separate peaks dominated by a broad asymmetric band in the $1100 \mathrm{~cm}^{-1}$ to $1750 \mathrm{~cm}^{-1}$ region centred at $1593.37 \mathrm{~cm}^{-1}$ (G-peak) and a shoulder centred at $1367.19 \mathrm{~cm}^{-1}$ (D-peak), as shown in Figure 4 . The intensity ratio of the D-peak to G-peak $\left(I_{\mathrm{D}} / I_{\mathrm{G}}\right)$ is 0.74 .

The G-peak is usually related to $\mathrm{sp}^{2} \mathrm{C}-\mathrm{C}$ stretching (aromatic or olefinic structures), while the D-peak is assigned to $\mathrm{sp}^{2}$ ordering between carbon atoms in a ring structure. 


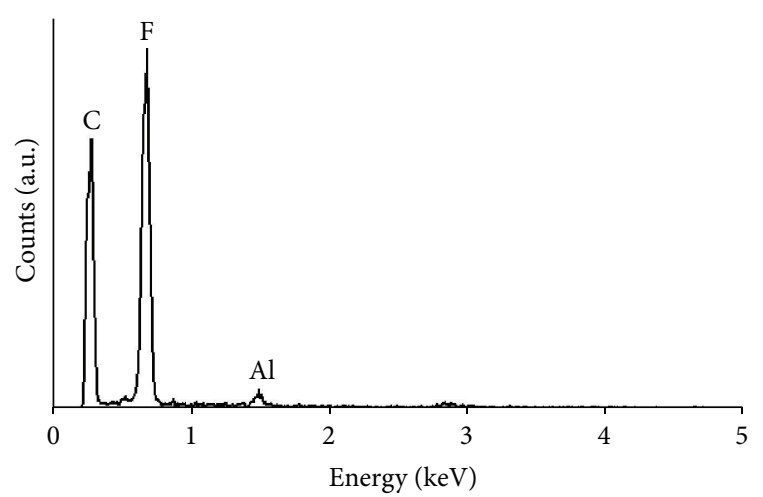

FIGURE 3: EDX signal of polymer redeposition adhering along the etched sidewall.

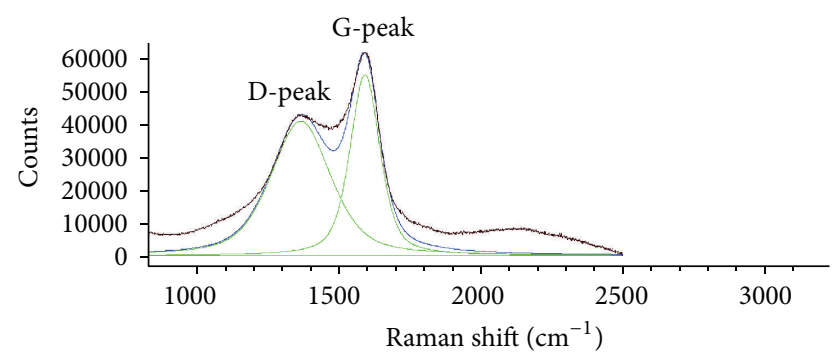

FIGURE 4: Raman spectrum of polymer redeposition. The locations of D-peak and G-peak are highlighted.

Zhu et al. [11] tried to produce a fluorinated amorphous carbon (a-C:F) film, which was prepared by using a magnetron cosputtering technique and used Teflon and graphite as targets. The prepared film was investigated in deep detail to assess the potential of a-C:F film being used for low $\kappa$ damascene multilevel interconnect applications. In this work, it was found that the prepared a-C:F film, which had a G-peak position at $1594 \mathrm{~cm}^{-1}$ and a ratio $\left(I_{\mathrm{D}} / I_{\mathrm{G}}\right)$ in the $0.49-0.70$ range, was characterised by Raman spectroscopy and had a fluorine concentration from $\sim 30 \mathrm{At} \%$ to $\sim 34 \mathrm{At} \%$. The data provided by Zhu is very close to our findings. Our results show that the prepared redeposition had a G-peak position at $1593.37 \mathrm{~cm}^{-1}$ and a ratio $\left(I_{\mathrm{D}} / I_{\mathrm{G}}\right)$ of 0.74 , which was identified by the Raman spectroscopy and had a fluorine concentration of $34.32 \mathrm{At} \%$. This evidence indicates that our redeposition was an a-C:F material.

3.3. Attenuated Total Reflected Infrared Spectroscopy. In Figure 5, the spectra show two strong absorption/reflection bands. A broad band in the region from $1230 \mathrm{~cm}^{-1}$ to $1570 \mathrm{~cm}^{-1}$ is associated with $-\mathrm{CF},-\mathrm{CF}_{2}$, and $-\mathrm{CF}_{3}$ stretching modes [12]. A broad band in the region from $1570 \mathrm{~cm}^{-1}$ to $1830 \mathrm{~cm}^{-1}$ and centred at $1730 \mathrm{~cm}^{-1}$ is associated with the presence of $\mathrm{C}=\mathrm{CF}_{2}$ group; these bands relate to $\mathrm{C}=\mathrm{C}$ stretching modes [13]. Finally, there is a broad band between $2890 \mathrm{~cm}^{-1}$ and $3680 \mathrm{~cm}^{-1}$ that is characteristic of $\mathrm{O}-\mathrm{H}$ stretching modes from absorbed water vapour. The water vapour is absorbed to the polymer redeposition surface due to the condensation
TABLE 1: Summary of $\%$ area fractions on Cls peak detected at the analysis points.

\begin{tabular}{lrcccccc}
\hline \multirow{2}{*}{ Point } & \multicolumn{9}{c}{ \% Area fraction } & \multirow{2}{*}{ \% Crosslink } \\
& $\mathrm{Ti}-\mathrm{C}$ & $\mathrm{C}-\mathrm{C}$ & $-\mathrm{C}-\mathrm{CF}_{x}$ & $-\mathrm{CF}$ & $-\mathrm{CF}_{2}$ & $-\mathrm{CF}_{3}$ & \\
\hline 1 & 2.09 & 14.77 & 19.21 & 29.22 & 24.61 & 10.30 & 64.34 \\
2 & 0.53 & 8.43 & 29.31 & 26.22 & 22.54 & 12.97 & 64.30 \\
\hline
\end{tabular}

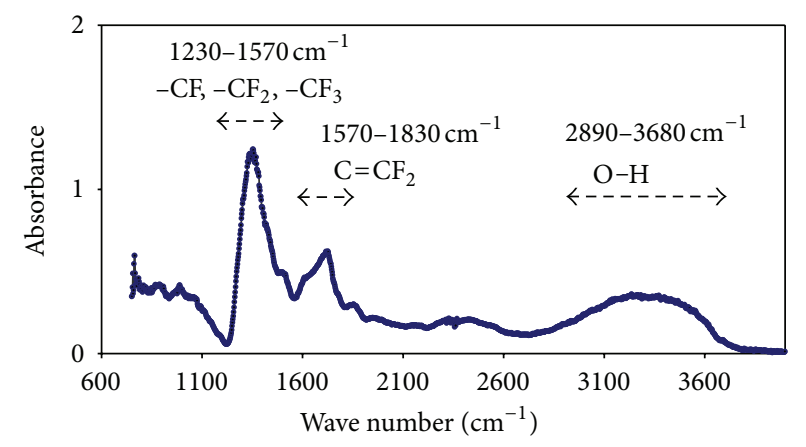

FIGURE 5: ATR FTIR spectrum of polymer redeposition.

occurring in the unloaded step of the RIE process because the temperature of the polymer redeposition in the chamber is cooler than room temperature.

3.4. X-Ray Photoelectron Spectroscopy. The results shown in Figures 6(a) and 6(b) refer to polymer redeposition at two analysis points, $4 \mathrm{~cm}$ apart, on the same sample, which were used to check the occurrence of polymer redeposition at the different locations. XPS analysis revealed that the polymer redeposition consisted of $-\mathrm{CF}_{3},-\mathrm{CF}_{2},-\mathrm{CF},-\mathrm{C}-\mathrm{CF}_{x}$, and $\mathrm{C}-\mathrm{C}$ bonds, which correspond to binding energies of $293.20 \mathrm{eV}, 291.05 \mathrm{eV}, 288.82 \mathrm{eV}, 286.44 \mathrm{eV}$, and $284.67 \mathrm{eV}$, respectively. A signal from a $\mathrm{Ti}-\mathrm{C}$ peak at a binding energy of $281.80 \mathrm{eV}$ was found on the analysed area but it was a small signal compared to the polymer peaks. Furthermore, the components were assembled in a random network as the nature of fluoropolymers is to have $64 \%$ crosslink at both points. The percentages of area fraction for each element of polymer redeposition are shown in Table 1. It was observed that the amounts of $\mathrm{C}-\mathrm{C}$ bond and $\mathrm{C}-\mathrm{CF}_{x}$ bond in the redeposition were significantly different between the two points: point \#1 had a C-C bond of $14.77 \%$ and a $-\mathrm{C}-\mathrm{CF}_{x}$ bond of $19.21 \%$, while point $\# 2$ had a C-C bond of $8.43 \%$ and a $-\mathrm{C}-\mathrm{CF}_{x}$ bond of $29.31 \%$. This difference is contributed by plasma uniformity: the plasma in the process chamber was not uniform between the two different processing points.

The equation to calculate the \%crosslink of a polymer is as follows:

\%Crosslink

$$
=\frac{\%(\mathrm{C}-\mathrm{C})+\%\left(-\mathrm{C}-\mathrm{CF}_{x}\right)+\%(-\mathrm{CF})}{100 \%-\%(\mathrm{Ti}-\mathrm{C})} \times 100 .
$$


The percentages of the crosslink of polymer redeposition were calculated from the total \% area fraction of the $\mathrm{C}-\mathrm{C}$, $\mathrm{C}-\mathrm{CF}_{x}$, and $-\mathrm{CF}$ peaks over the total $\%$ area fraction (after subtraction of the signal of $\mathrm{Ti}-\mathrm{C}$ ).

It is important to note here that there are some conditions that can provide polymer redeposition in this process window. The etching conditions should not allow too much of an etching mechanism or polymer deposition. In case the test etching conditions provide too much of an etching mechanism, the redeposition would be in the $\mathrm{AlF}_{x}$ form due to the plasma containing abundant $\mathrm{F}$ species that are responsible for etching the substrate. In our DOE result using $\mathrm{CF}_{4}$ gas and both levels (low and high) of coil power and gas flow rate settings, it provided a high etching mechanism and then $\mathrm{AlF}_{x}$ redeposition took place. For etching conditions providing too much polymer deposition, the AlTiC would not be etched but would generate a polymer that is deposited directly on the AlTiC surface due to the plasma containing abundant $\mathrm{C}_{x} \mathrm{~F}_{y}$ species that are responsible for the deposition mechanism. In our DOE result using $\mathrm{C}_{4} \mathrm{~F}_{8}$ gas at a low coil power setting $(300 \mathrm{~W}-400 \mathrm{~W})$ with both levels of gas flow rate settings, it provided a high polymer deposition mechanism but AlTiC was not etched. The optimal conditions using $\mathrm{C}_{4} \mathrm{~F}_{8}$ gas were a coil power of $700 \mathrm{~W}$ and a gas flow rate of $14 \mathrm{sccm}$. This process regime provided a balance between the etching and the deposition mechanism, eventually allowing redeposition in the polymer mode. The key of this concept is to modify the $\mathrm{AlF}_{x}$ redeposition to the polymer redeposition. The plasma should contain sufficient polymer species $\left(\mathrm{CF}_{x}\right)$ to deposit directly on a sidewall during $\mathrm{F}$ species etching to the substrate; the redeposition on the etched sidewall will then contain greater $\mathrm{CF}_{x}$ than $\mathrm{AlF}_{x}$.

To get the correct chemical bonding data of the redeposition material, both Raman and FTIR spectra must be considered: Raman spectroscopy analyses the vibration and rotational modes of molecules using the inelastic scattering of light and infrared spectroscopy analyses the amount of light that molecules absorb to determine the vibration and rotational modes of molecules. The XPS spectra results in Figures 6(a) and 6(b) indicate that there was $-\mathrm{CF},-\mathrm{CF}_{2}$, and $-\mathrm{CF}_{3}$ bonding present in the redeposition material. These bonding types are also present in FTIR spectra $\left(1230 \mathrm{~cm}^{-1}-1570 \mathrm{~cm}^{-1}\right)$ and Raman spectra $\left(1200 \mathrm{~cm}^{-1}-1500 \mathrm{~cm}^{-1}\right.$, D-peak). Moreover, FTIR should detect $\mathrm{C} / \mathrm{F}$ complex bonding, $\mathrm{C}=\mathrm{CF}_{2}$ and $\mathrm{C}=\mathrm{C}$, in the spectra region $1570 \mathrm{~cm}^{-1}-1830 \mathrm{~cm}^{-1}$, corresponding to a Raman spectra at the G-peak of $1593.37 \mathrm{~cm}^{-1}$, assigned to $-\mathrm{C}=\mathrm{C}-\left(\mathrm{sp}^{2} \mathrm{C}-\mathrm{C}\right)$ in stretching mode. The XPS data confirmed the existence of these bonding types.

However, in this paper, we also discuss whether $\operatorname{AlF}_{x}(\mathrm{~s})$ is formed in the C-F redeposition bulk. Regarding the ATRFTIR data, there are many centre peak positions of Al-F vibration bonding $[14,15]$ and the major peaks are at $790 \mathrm{~cm}^{-1}$ $\left(750-858 \mathrm{~cm}^{-1}\right), 960 \mathrm{~cm}^{-1}\left(856-1122 \mathrm{~cm}^{-1}\right), 1460 \mathrm{~cm}^{-1}(1432-$ $\left.1488 \mathrm{~cm}^{-1}\right), 1640 \mathrm{~cm}^{-1}\left(1602-1662 \mathrm{~cm}^{-1}\right), 1741 \mathrm{~cm}^{-1}(1710-$ $\left.1768 \mathrm{~cm}^{-1}\right)$, and $2364 \mathrm{~cm}^{-1}\left(2352-2385 \mathrm{~cm}^{-1}\right)$, in which the number in the bracket is the integrated area intensity. In this paper, there are no FTIR positions of Al-F vibration at $1460 \mathrm{~cm}^{-1}, 1640 \mathrm{~cm}^{-1}$, and $1741^{-1}$ as FTIR can only detect

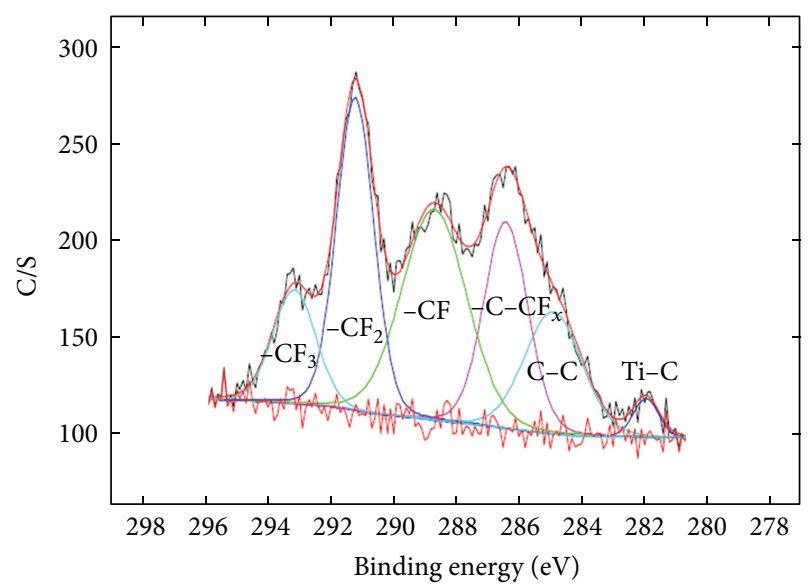

(a)

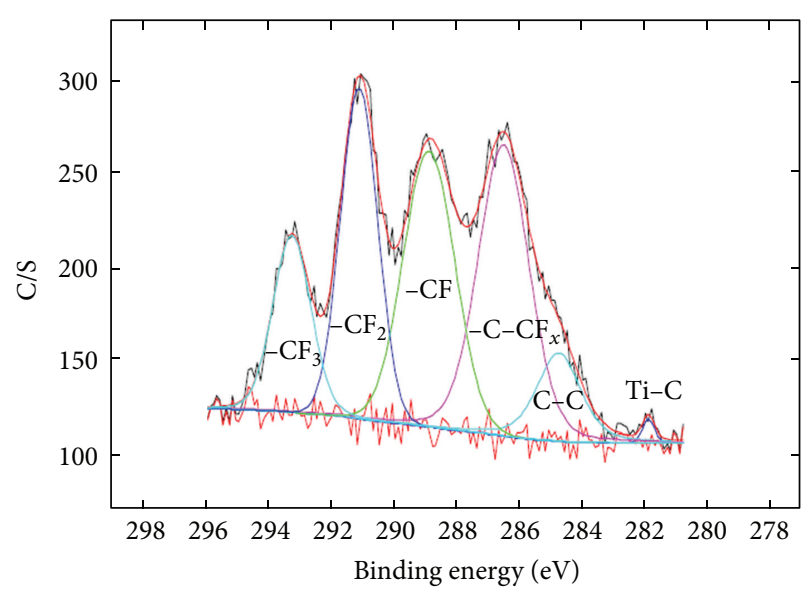

(b)

FIGURE 6: XPS spectrum of polymer redeposition at point \#1 (a) and point \#2 (b).

the higher signals and the absorbance of the C-F peak is higher than the absorbance of Al-F. So, they overlapped at $1230-1570 \mathrm{~cm}^{-1}\left(-\mathrm{CF},-\mathrm{CF}_{2}\right.$, and $\left.-\mathrm{CF}_{3}\right)$ and $1570-1830 \mathrm{~cm}^{-1}$ $\left(\mathrm{C}=\mathrm{CF}_{2}\right)$ (Figure 5), respectively. The EDX data (Figure 3) shows the high intensity of the C-peak and the F-peak, which is in line with the FTIR data (Figure 5). Hence, the band positions appearing at $1230-1570 \mathrm{~cm}^{-1}$ and $1570-1830 \mathrm{~cm}^{-1}$ should belong to the $\mathrm{C}-\mathrm{F}$ bonding. The intensities of the $\mathrm{Al}-\mathrm{F}$ bond positions at $790 \mathrm{~cm}^{-1}, 960 \mathrm{~cm}^{-1}$, and $2364 \mathrm{~cm}^{-1}$ are very low so it is reasonable for considering these as noise and for considering that the Al-F bond does not exist in the C-F redeposition material. So, we can conclude here that the redeposition bulk is the $\mathrm{C}-\mathrm{F}$ polymer-rich redeposition.

We successfully demonstrated the modification of redeposition from an $\mathrm{AlF}_{3}$ redeposition, which is difficult to remove with an IPA-based solution during the resist strip process, to a C-F polymer redeposition, which is easy to remove in this step. Furthermore, polymer redeposition that is fluorocarbon based can be destroyed by the reaction of $\mathrm{OH}$ radicals that are a component of the IPA molecule. 
The reaction between fluorocarbon $\mathrm{CF}_{2}$ and $\mathrm{OH}$ radicals is confirmed by the work of Srinivasan [16].

\section{Conclusions}

The EDX signal generated from the SEM analysis indicates that the polymer redeposition contained carbon atoms (63.70 At\%) and fluorine atoms (34.32 At\%). A Raman spectrum analysis of the polymer redeposition shows a broad asymmetric band in the $1100 \mathrm{~cm}^{-1}$ to $1750 \mathrm{~cm}^{-1}$ region centred at $1593.37 \mathrm{~cm}^{-1}$ (G-peak), which relates to a C-C group in the aromatic and olefinic structure and a shoulder centred at $1367.19 \mathrm{~cm}^{-1}$ (D-peak), related to carbon atoms in the ring structure. The FTIR spectra reveal that the polymer redeposition is associated with $-\mathrm{CF},-\mathrm{CF}_{2},-\mathrm{CF}_{3}$, and also $\mathrm{C}=\mathrm{CF}_{2}$ groups. The XPS analysis indicates that the polymer redeposition consists of $-\mathrm{CF}_{3},-\mathrm{CF}_{2},-\mathrm{CF},-\mathrm{C}-\mathrm{CF}_{x}$, and $\mathrm{C}-\mathrm{C}$ assembled in a random network with $64 \%$ crosslink.

All the characterised results of the EDX, Raman, FTIR and XPS analyses indicate that the polymer redeposition is a fluoropolymer redeposition.

\section{Acknowledgments}

The authors wish to acknowledge the financial support from the Industry/University Cooperative Research Center in Data Storage Technology and Applications, King Mongkut's Institute of Technology Ladkrabang, and the National Electronics and Computer Technology Center, National Science and Technology Development Agency. Moreover, they would like to thank Mark Moravec for his help in reviewing this paper and also Miss Alonggot Limcharoen for her help in preparing this paper. The authors would like to thank the Commission of Higher Education under the National Research University (NRU) Project.

\section{References}

[1] R. Hsiao, "Fabrication of magnetic recording heads and dry etching of head materials," IBM Journal of Research and Development, vol. 43, no. 1-2, pp. 89-102, 1999.

[2] M. Zhang, Y. S. Hor, G. Han, and B. Liu, "Dry etching of AlTiC with $\mathrm{CF}_{4}$ and $\mathrm{H}_{2}$ for slider fabrication," IEEE Transactions on Magnetics, vol. 39, no. 5, pp. 2486-2488, 2003.

[3] J. W. Kim, Y. C. Kim, and W. J. Lee, "Reactive ion etching mechanism of plasma enhanced chemically vapor deposited aluminum oxide film in $\mathrm{CF}_{4} / \mathrm{O}_{2}$ plasma," Journal of Applied Physics, vol. 78, no. 3, pp. 2045-2049, 1995.

[4] B. Bhushan and N. S. Tambe, "Role of particulate contamination on friction and wear and durability of load/unload and padded picosliders," IEEE Transactions on Magnetics, vol. 39, no. 2, pp. 857-863, 2003.

[5] J. Bhardwaj, D. M. Haynes, J. A. Grange et al., "High-throughput inductively coupled plasma production platform for etching airbearing surface on GMR read/write heads," in Datateched, pp. 131-140, 1st edition, 1998.

[6] N. Fukushima, H. Katai, T. Wada, and Y. Horiike, "High-rate and smooth surface etching of $\mathrm{Al}_{2} \mathrm{O}_{3}$-TiC employing Inductively Coupled Plasma (ICP)," Japanese Journal of Applied Physics, vol. 35, no. 4, part 1, pp. 2512-2515, 1996.
[7] J. W. Coburn, "In situ Auger electron spectroscopy of $\mathrm{Si}$ and $\mathrm{SiO}_{2}$ surfaces plasma etched in $\mathrm{CF}_{4}-\mathrm{H}_{2}$ glow discharges," Journal of Applied Physics, vol. 50, no. 8, pp. 5210-5213, 1979.

[8] J. W. Coburn and H. F. Winters, "Ion- and electron-assisted gas-surface chemistry-an important effect in plasma etching," Journal of Applied Physics, vol. 50, no. 5, pp. 3189-3196, 1979.

[9] B. Ji, S. A. Motika, P. R. Badowski, S. Dheandhanoo, J. R. Stets, and E. J. Karwacki, "Fluorine plasma chemistry for high-AR dielectric etching," Solid State Technology, vol. 48, no. 11, pp. 4552, 2005.

[10] I. R. Johnston, H. Ashraf, J. K. Bhardwaj et al., "Etching 200-mm diameter SCALPEL mask with the ASE process," in Emerging Lithographic Technologies IV, vol. 3997 of Proceeding of SPIE, pp. 184-193, July 2000.

[11] W. Zhu, C. S. Pai, H. E. Bair et al., "Novel co-sputtered fluorinated amorphous carbon films for sub- $0.25 \mu \mathrm{m}$ low $\kappa$ damascene multilevel interconnect applications," in Proceedings of the Electron Devices Meeting (IEDM '98), pp. 845-848, December 1998.

[12] C. E. Bottani, A. Lamperti, L. Nobili, and P. M. Ossi, "Structure and mechanical properties of PACVD fluorinated amorphous carbon films," Thin Solid Films, vol. 433, no. 1-2, pp. 149-154, 2003.

[13] F. L. Freire Jr., M. E. H. Maia da Costa, L. G. Jacobsohn, and D. F. Franceschini, "Film growth and relationship between microstructure and mechanical properties of a-C:H:F films deposited by PECVD," Diamond and Related Materials, vol. 10, no. 2, pp. 125-131, 2001.

[14] C. Alonso, A. Morato, F. Medina et al., "Preparation and characterization of different phases of aluminum trifluoride," Chemistry of Materials, vol. 12, no. 4, pp. 1148-1155, 2000.

[15] S. Saito, K. Sugita, and J. Tonotani, "Effect of $\mathrm{CHF}_{3}$ addition on reactive ion etching of aluminum using inductively coupled plasma," Japanese Journal of Applied Physics, vol. 44, no. 5, pp. 2971-2975, 2005.

[16] N. K. Srinivasan, M. C. Su, J. V. Michael, A. W. Jasper, S. J. Klippenstein, and L. B. Harding, "Thermal decomposition of $\mathrm{CF}_{3}$ and the reaction of $\mathrm{CF}_{2}+\mathrm{OH} \rightarrow \mathrm{CF}_{2} \mathrm{O}+\mathrm{H}$," Journal of Physical Chemistry A, vol. 112, no. 1, pp. 31-37, 2008. 

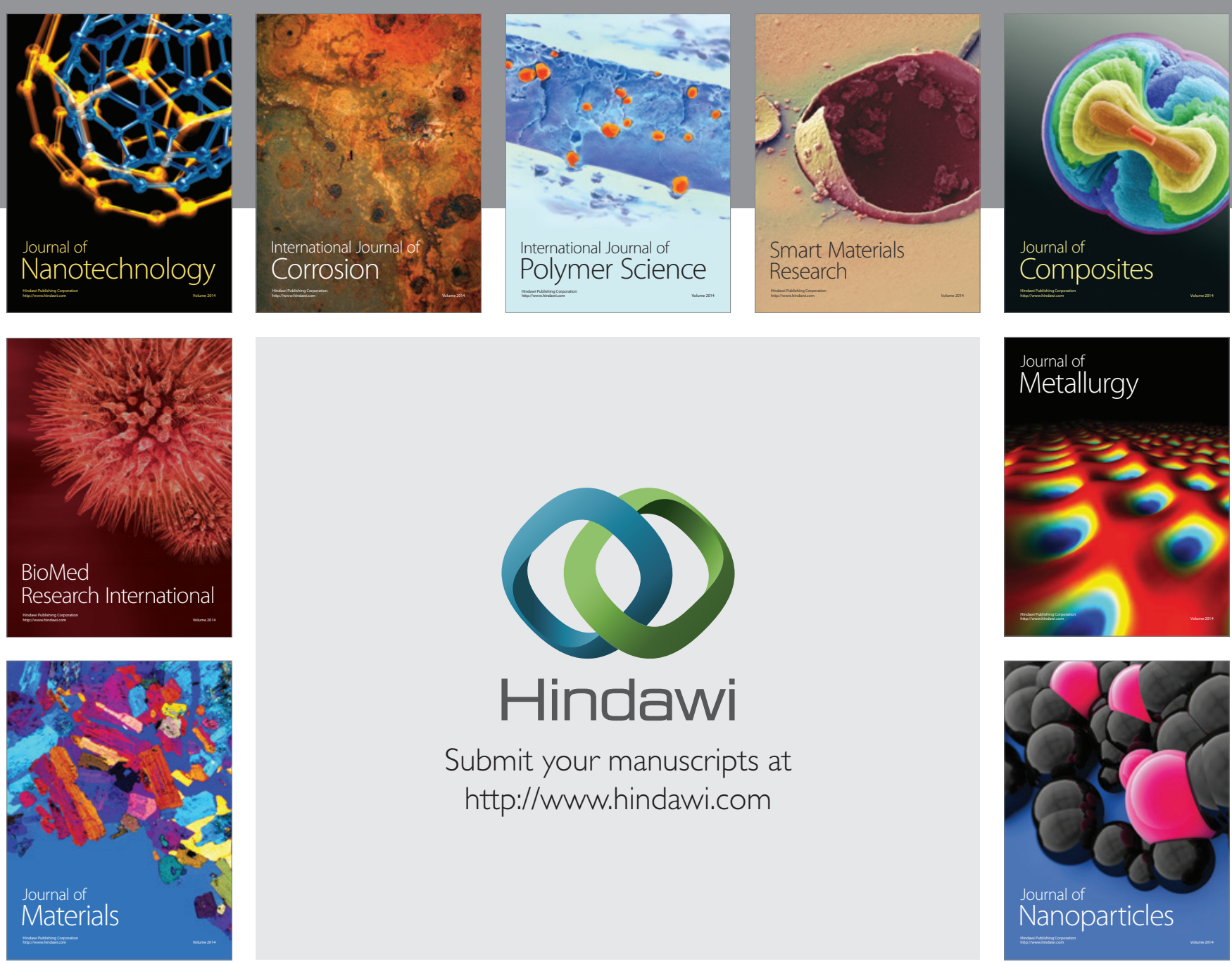

Submit your manuscripts at http://www.hindawi.com
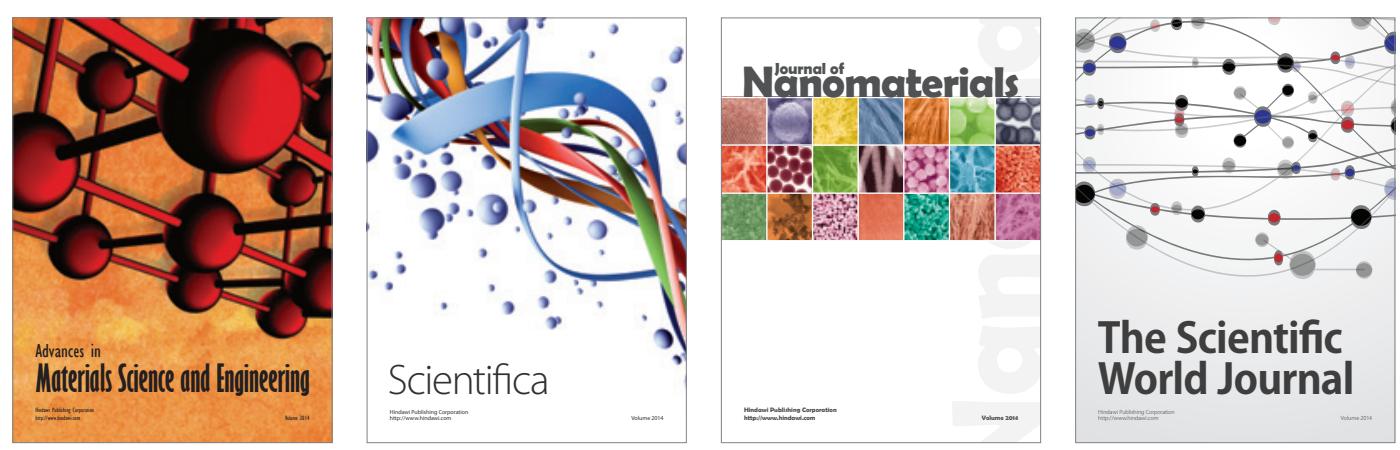

\section{The Scientific World Journal}
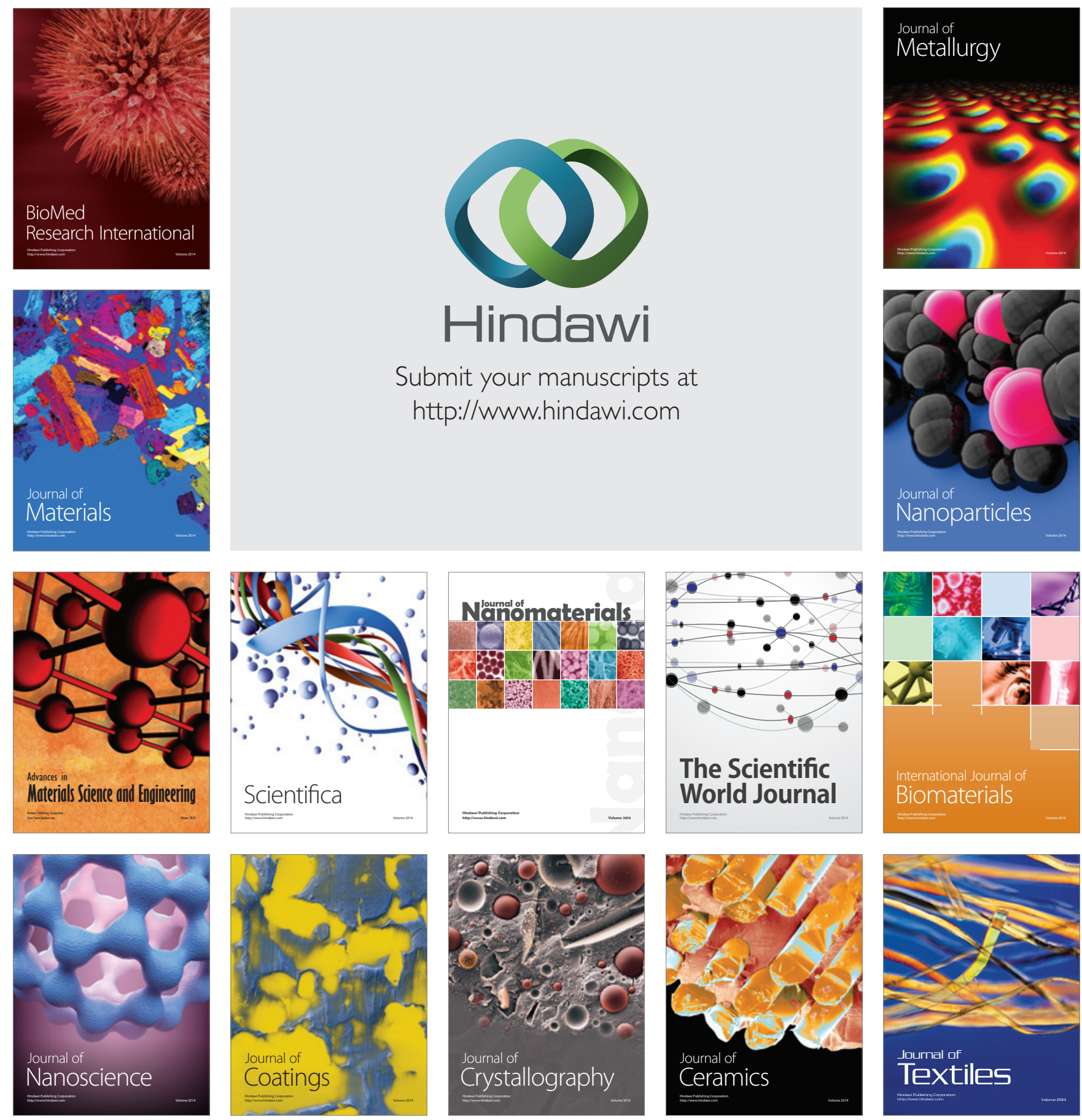